\title{
A relação entre Dislipidemia e Diabetes Mellitus tipo 2
}

\section{The relation between Dyslipidemia and Diabetes Mellitus type 2}

\section{Renata Pereira1}

Artigo

Original

Original

Paper

Palavras-chave:

Dislipidemia

Diabéticos mellitus tipo 2

Obesidade

Doenças

cardiovasculares
Resumo:

O diabetes mellitus tipo 2 (DM2) é uma doença crônica caracterizada por defeitos da ação e na secreção da insulina, podendo ocorrer em indivíduos de qualquer idade, mas pode ser diagnosticado em idades mais avançadas. A obesidade e o DM2 são doenças que causam resistência à insulina, caracterizada pelo aumento na produção de glicose, hipertrigliceridemia e lipoproteína de muito baixa densidade (VLDL), associados à redução da lipoproteína de alta densidade (HDL) e elevação na lipoproteína de baixa densidade (LDL). A aterosclerose possui um dos principais fatores de riscos a dislipidemia que é causada pela elevação de colesterol na corrente sanguínea. A melhor maneira de tratar e prevenir a dislipidemia e os DM2 é praticando atividades físicas, obtendo hábitos alimentares mais saudáveis e fazendo o uso de medicamentos adequados. O presente estudo teve como objetivo de estabelecer relações entre a dislipidemia e a DM2.

\section{Abstract}

Diabetes mellitus type 2 (T2DM) is a chronic disease characterized by defects in the action and secretion of insulin and it can occur in individuals of any age, but can be diagnosed at older ages. Obesity and type 2 diabetes are diseases that cause insulin resistance, characterized by increased production of glucose, hypertriglyceridemia, very low density lipoprotein (VLDL) associated with reduced high density lipoprotein $(H D L)$ and elevated in low density lipoprotein (LDL). Atherosclerosis has one of the main risk factors for dyslipidemia which is caused by the elevation of cholesterol in the bloodstream. The best way of treating or preventing dyslipidemia and T2DM is practicing physical activity, getting healthier eating habits and making use of drugs. This study aimed to establish relation between dyslipidemia and T2DM.
Key words:

Dyslipidemia

Diabetes mellitus type 2

Obesity

Cardiovascular diseases 


\section{Introdução}

Atualmente as doenças cardiovasculares e a aterosclerose são consideradas um dos principais problemas da saúde pública no Brasil e no mundo, podendo levar o indivíduo a óbito com idades cada vez mais precoces. Os pacientes com DM2 apresentam dislipidemia que é a elevação das taxas de lipídios e de lipoproteínas na corrente sanguínea, ocasionando posteriormente as doenças cardiovasculares. Geralmente a dislipidemia está presente no momento do diagnóstico da hiperglicemia nas pessoas com DM2 (SCHEFFEL et al., 2004; SCHAAN, HARZHEIM e GUS, 2004 e WAJCHENBERG et al., 2007).

Os maus hábitos alimentares, o sedentarismo e história familiar de doenças coronarianas influenciam nas alterações das lipoproteínas plasmática circulantes no sangue, podendo levar o indivíduo a sofrer arteriosclerose e doenças cardiovasculares. A dislipidemia está relacionada pelo meio em que o indivíduo vive e pelos fatores genéticos.

Lipoproteínas são estruturas macromoleculares que facilitam o metabolismo lipídico e o transporte de lipídeos na circulação. As lipoproteínas são classificadas da seguinte maneira: lipoproteína de alta densidade (HDL), lipoproteína de baixa densidade (LDL), lipoproteína de densidade intermediária (IDL), lipoproteína de densidade muito baixa (VLDL), e quilomicrons (HARIS, 2007). Nos DM2 os níveis de triglicerídeos elevam e os níveis de HDL diminuem.

Pitanga (2001), em seu trabalho, afirmou que a prática de atividades físicas é importante porque influência nos valores mais favoráveis das lipoproteínas plasmáticas e para a prevenção de doenças cardiovasculares, dislipidemia e DM2.

O presente artigo se tratou de uma revisão de literatura baseada em livros, e artigos científicos que abrange os anos de 2001 a 2009. Os artigos foram procurados no site do SCIELO Scientific Electronic Library Online (Biblioteca Científica Eletrônica em Linha) que é uma cooperativa de publicação de periódicos científicos na internet. Para a coleta de artigos foram utilizadas as seguintes palavras-chave: Dislipidemia, diabetes tipo 2, obesidade e doenças cardiovasculares. Com o aumento de pessoas com DM2, obesidade e dislipidemia, viu-se a necessidade de realizar o presente estudo que teve o objetivo de estabelecer a relação entre a dislipidemia e a DM2, através da revisão de literatura.

\section{Diabetes Mellitus tipo 2 (DM2) e Obesidade}

O DM2 é uma doença caracterizada por defeitos da ação e na secreção da insulina, podendo ocorrer em qualquer idade, mas geralmente é diagnosticada após dos 40 anos. A maioria das pessoas que tem DM2 é obesa ou tem sobre peso e são insulino-independentes, mas podem necessitar de tratamento com insulina para a obtenção de um controle metabólico adequado (DIRETRIZES SBD, 2006). A insulina que é produzida nos DM2 não é suficiente para controlar a produção de glicose pelo fígado ou promover capacitação de glicose pelo músculo esquelético (GLEW e CRABB, 2007). A doença aparece lentamente e os sintomas iniciais não são reconhecidos. (LEHNINGER, 2006)

Nas últimas décadas a importância da diabetes vem crescendo em decorrência de maior taxa de urbanização, aumento da expectativa de vida, industrialização, aumento do consumo de dietas hipercalóricas, deslocamento da população para zonas urbanas, mudança de estilos de vida tradicionais para modernos, sedentarismo e obesidade (ORTIZ, 2001).

A obesidade e o DM2 são doenças que causam resistência à insulina. $\mathrm{Na}$ obesidade, em geral a quantidade de gordura corporal é proporcional ao grau de resistência à insulina. Nestes indivíduos, os níveis de insulina são altos no plasma e com isso, favorecerá no desenvolvimento da DM2. A obesidade deve ser tratada para que a síndrome metabólica, o DM2 e a doença cardiovascular não progridam. (GLEW e CRABB, 2007). A prevalência da obesidade segundo Correa et al. (2003), aumentou $100 \%$ nas ultimas décadas, tendo como com consequência aumento da incidência destas comorbidades.

Silveira constatou que a correlação de obesidade e DM2 existem, mas não é muito alta, se o indivíduo aumentar a sua gordura corporal, os níveis glicêmicos também será elevados e com isso, pode aumentar o risco de 
desenvolver DM2. E observou que nos seus resultados o aumento da glicemia, está relacionado com a elevação do valor do índice de massa corpórea (IMC) que é um fato relacionado ao aumento da resistência à insulina.

\section{Dislipidemia}

Com a produção desregulada de colesterol no organismo dos seres humanos, pode acarretar a sérios problemas, como de obstrução das paredes do vaso sanguíneo, denominado aterosclerose que está ligada ao aumento do colesterol no sangue (LEHNINGER, 2006).

As doenças ateroscleróticas vêm causando mortalidade no Brasil, tendo a dislipidemia como um dos principais fatores de riscos (SANTOS, D.R., 2001).

A dislipidemia vem de forma silenciosa na infância e só se desenvolve na vida adulta, mas isso depende do ritmo e do estilo de vida que a pessoa leva (FARIA; DALPINO e TAKATA, 2008).

Kolankiewicz; Giovelli e Bellinaso (2008), em um estudo cujo objetivo era conhecer e analisar o perfil lipídico e determinar a prevalência de dislipidemias em indivíduos adultos concluiu que os indivíduos que tem risco de ter doença aterosclerótica não só os diabéticos, também os hipertensos, sedentários, obesos, os fumantes e indivíduos que tem histórico familiar desta doença.

A Segundo Diretrizes da Sociedade Brasileira de Diabetes os pacientes com aterosclerose DM2 estão sujeitos de duas a quatro vezes mais risco para doenças cardiovasculares (DCV) quando comparados a pacientes não diabéticos. Os indivíduos com hipertensão arterial e diabetes mellitus apresentaram maior prevalência de dislipidemias. (SOUZA et al., 2003)

Etiologicamente a dislipidemia é classificada como dislipidemia primária que é de origem genética que é causada pela desordem da síntese e degradação de lipídios, e dislipidemia secundária que é causada por outras doenças ou uso de medicamento. O DM2 e a obesidade são classificados como dislipidemia secundária (SANTOS, D.R., 2001).

\section{Perfil lipídico dos DM2 com dislipidemia}

A dislipidemia é uma alteração do metabolismo de lipoproteínas circulantes no sangue que nos DM2 é causada devido à resistência a insulina e a obesidade, podendo ser caracterizada pelo aumento na produção de glicose, hipertrigliceridemia e VLDL, associando-se a redução na HDL e elevação na LDL (que apresenta partícula pequena e densa). Isso é devido ao excesso de ácidos graxos circulantes derivados do tecido adiposo encontrado no fígado. Com produção excessiva de ácidos graxos, há redução da sensibilidade à insulina no tecido muscular, há produção de interleucinas, fatores de crescimento e outras citosinas pelo tecido adiposo e também pode há hiperinsulinemia que aumenta a reabsorção de sódio e a atividade do sistema simpático. A média das concentrações comparada com os pacientes não diabéticos de LDL, apresenta diferença qualitativa por perfil de elevada aterogenicidade através de maior proporção das partículas de LDL, mas qualitativamente não apresenta diferença. Com os triglicerídeos elevados, pode resultar o decréscimo da lipólise dos triglicerídeos presentes nas partículas de VLDL (SANTOS, D.R., 2001 E DIRETRIZ SBD, 2006). Adiels et al. (2005) afirma que o DM2 e resistência à insulina estão associados com excesso de produção hepática de VLDL.

O estudo de Vasques et al. (2009) teve o objetivo de investigar a habilidade de indicadores bioquímicos do perfil lipídico plasmático em identificar resistência à insulina, avaliada pelo índice HOMA-IR (Homeostasis Model Assessment - Insulin Resistance), avaliaram 138 homens saudáveis com idade 20-59 anos e analisaram os indicadores bioquímicos do perfil lipídico que são: triglicérides, colesterol total, HDL, LDL, e as relações colesterol total /HDL e triglicerídeos/HDL, observaram que a relação triglicerídeos/HDL apresentou a correlação mais forte e a maior, pois tem boa habilidade de identificar a resistência à insulina na prática clinica já o colesterol total e LDL, não apresentaram resultados estatísticos significantes.

Naheed et al. (2003) conheceram o padrão de dislipidemia entre os DM2 e observaram que o colesterol total e LDL estavam elevados e concluíram que os pacientes devem 
ser instruídos a verificar regularmente os lipídios anormais para que seja feito o controle do açúcar e de lipídeos de maneira eficaz. E afirmou que a dislipidemia nos DM2 é uma importante causa de mordilidade e pode aumentar o risco de doença coronária.

Foram observados em um estudo realizado em Campos dos Goytacazes, nos Estado do Rio de Janeiro os indivíduos com dislipidemia ou com hipertensão tem riscos três vezes maior de desenvolver o DM2 em comparação aos indivíduos sem fatores de riscos e a prevalência da DM2 (SOUZA, 2003).

Santos, Balzaneli e D'Andrade (2009) verificou perfil lipídico de pacientes diabéticos e hipertensos tratados com a combinação do captopril com hidroclorotiazida tem efeito protetor e levou a menores níveis de triglicerídeos e níveis de HDL ligeiramente elevados, diminuindo o risco aterosclerótico dos pacientes e minimizando o efeito deletério aterogênico da hidroclorotiazida.

\section{Tratamento e prevenção}

De uma maneira geral, para tratar e prevenir não só a dislipidemia, mas também a obesidade, e o DM2, o indivíduo deve praticar atividades físicas, e obter hábitos alimentares mais saudáveis, evitar o fumo e bebidas alcoólicas. Deve-se fazer o uso do medicamento para o tratamento. Tem casos que a cirurgia para emagrecer é eficaz. Conforme a III Diretrizes Brasileiras sobre dislipidemias e diretriz de prevenção da aterosclerose, nos DM2 o controle da dislipidemia passa pelo controle da glicemia e do uso de medicamentos específicos dependendo da necessidade do paciente.

Lyra et al. (2006) afirmou em seu trabalho que a cirurgia bariátrica realizada em obesos mórbidos pode ser eficaz para a redução no desenvolvimento do DM2.

Segundo o Consenso Brasileiro Sobre Dislipidemias da Sociedade Brasileira de Cardiologia, os medicamentos possuem diferentes mecanismos de ação. Os medicamentos usados para o tratamento da dislipidemia no Brasil são: Fibratos, Ácidos Nicotínicos e Derivados, Sequestrastes de Ácidos Biliares, Probucol, Ácidos Graxos Ômega-3 e Vastatinas.
No seu trabalho, Silva e Lima (2002) afirmaram que é importante o exercício físico, pelo menos quatro vezes na semana, com seções de 1 hora para os DM2 e com isso diminui os triglicerídeos, aumenta o HDL, diminui a frequência cardíaca de repouso e também pode melhorar a glicemia de jejum e a hemoglobina glicosilada.

Segundo IV Diretriz Brasileira Sobre Dislipidemias e Prevenção da Aterosclerose para tratar da hipertrigliceridemia secundária nos diabéticos, a dieta deve ser hipocalórica, o consumo de bebida alcoólica tem restrição total e o consumo de carboidratos e gordura deverá sofrer adequação. A atividade física, praticada regularmente, ajuda a controle das dislipidemias e doença arterial coronária. Os exercícios aeróbios como caminhada, corrida, ciclismo, natação, promove a redução dos triglicerídeos, aumenta os níveis de HDL, e o índice de LDL não sofre alteração significativas. Para a realização de atividade o indivíduo deve passar por uma a avaliação clinica e deve ser realizado um teste ergométrico ou fazer o teste cardiorrespiratório em esforço (ergoespirometria) para determinação da sua capacidade física.

\section{Conclusão}

O DM2 e a dislipidemia têm grandes relações, pois quanto mais gordura no organismo, mais aumenta o nível de glicose e de lipídeos no sangue. Isso leva ao desenvolvimento de doenças cardiovasculares que vêm causando mortalidades em milhares de pessoas no mundo. Este trabalho buscou a destacar a importância da relação entre a dislipidemia e o DM2 no intuito de alertar o risco de doenças cardiovasculares e aterosclerose. O levantamento dos artigos referente à dislipidemia, doenças cardiovasculares e DM2 mostrou que a obesidade tem grande relação a essas doenças. Existe a necessidade de mais estudos nesta área para melhorar e amenizar a dislipidemia e a DM2.

É importante que haja um esclarecimento necessário para as pessoas sobre a alimentação saudável, e a prática regular de atividades física como medida preventiva. Trabalhos educativos nas escolas e comunidades voltados para prevenção, diagnóstico e tratamento deveriam ser realizados por profissionais especializados. 
7. Referências bibliográficas

1. GLEW, R.A.; CRABB, D.W. Interrelações metabólicas. In: DEVLIN, T.M.; MICHELACCI, Y.M. Manual de Bioquímica com Correlações Clínicas. São Paulo: Editora Blücher, 2007. P. 829-869.

2. HARRIS, R.H. Metabolismo de Lipídeos: Vias do metabolismo de lipídeos especiais. In: DEVLIN, T.M.; MICHELACCI, Y.M. Manual de Bioquímica com Correlações Clínicas. São Paulo: Editora Blücher, 2007. P. 683-724.

3. LEHNINGER, A.L. Princípios de Bioquímica. 4ª Ed. SP: Sarvier, 2006.

4. SCHEFFEL, R. S. et al. Prevalência de complicações micro e macrovasculares e de seus fatores de risco em pacientes com Diabetes Melito do Tipo 2 em atendimento ambulatorial. Revista da Associação Médica Brasileira. V. 50, n. 3, p.263-267; 2004.

5. SCHAAN, B. D.A.; HARZHEIM, E.; GUS, I. Perfil de risco cardíaco no diabetes mellitus e na glicemia de jejum alterada. Revista de Saúde Pública. V. 38, n. 4, p. 529-536; 2004.

6. WAJCHENBERG,B.L.etal.Posicionamento oficial da $\mathrm{SBD}^{\circ}$ 5. Dislipidemia no paciente diabético: aspectos etiopatogênicos, clínicos e terapêuticos. Revista Brasileira de Medicina, suplemento especial 5/2007.

7. PITANGA F.J.G. Atividade física e lipoproteínas plasmáticas em adultos de ambos os sexos. Revista Brasileira de Ciências e Movimento. Brasília. V. 9 n. 4, p. 25-31, outubro 2001

8. DIRETRIZES SBD, Diabetes mellitus Tratamento e acompanhamento das Diretrizes da Sociedade Brasileira de Diabetes, 153 p., 2006.

9. ORTIZ, M. C. A. e ZANETTI M. L. Levantamento dos fatores de risco para Diabetes Mellitus Tipo 2 em uma instituição de Ensino Superior. Revista Latino-americana de Enfermagem. V. 9, n. 3, p. 58-63; maio, 2001.
10. CORRÊA, F. H.S. et al. Influência da Gordura Corporal no Controle Clínico e Metabólico de Pacientes Com Diabetes Mellitus Tipo 2. Arquivos Brasileiros de Endocrinologia \& Metabologia. V. 47, n. 1, fevereiro, 2003.

11. SILVEIRA, L. A. G Correlação entre Obesidade e Diabetes Tipo 2. Pósgraduação Latu-Sensu em Fisiologia do Exercício e Avaliação-Morfofuncional Universidade Gama Filho. Juiz de Fora.

12. SANTOS, D.R. III Diretrizes Brasileiras sobreDislipidemiaseDiretrizdePrevenção da Aterosclerose do Departamento de Aterosclerose da Sociedade Brasileira de Cardiologia. Arquivos Brasileiros de Cardiologia ; 77 (S3): 1-48, 2001.

13. KOLANKIEWICZ, F; GIOVELLI, F.M.H.; BELLINASOM.L.B. Estudo do perfil lipídico e da prevalência de dislipidemias em adultos. RBAC. V. 40, n. 4, p. 317-320, 2008.

14. SOUZA, L.J. et al. Prevalência de Dislipidemia e Fatores de Risco em Campos dos Goytacazes - RJ. Arquivos Brasileiros de Cardiologia. V. 81, n. 3, p. 249-56, 2003.

15. ADIELS, M. Overproduction of VLDL 1 Driven by Hyperglycemia Is a Dominant Feature of Diabetic Dyslipidemia. Arteriosclerosis, Thrombosis, and Vascular Biology. 25:1697, 2005.

16. VASQUES, A.C.J. Indicadores do perfil lipídicoplasmáticorelacionadoàresistência à insulina. Revista da Associação Médica Brasileira. V. 55, n. 3, p. 342-346, 2009.

17. NAHEED, T. et al. Dyslipidemias in Type II Diabetes Mellitus Patients in a teaching Hospital of Lahore, Pakistan. Pakistan Journal of Medical Sciences. V. 19, n.4, p- 283-286, 2003.

18. SOUZA, L.J. Prevalência de Diabetes Mellitus e Fatores de Risco em Campos dos Goytacazes, RJ. Arquivos Brasileiros de Endocrinologia \& Metabologia. V. 47 n. 1 Fevereiro 2003 
19. SANTOS, F.B.F; BALZANELI, E.S. e ANDRADE, M.R.P.D. Avaliação do perfil lipídico de pacientes diabéticos e hipertensos tratados com captopril. Jornal Brasileiro de Patologia e Medicina Laboratorial. V. 45, n. 3, p. 207-212, junho 2009.

20. SEGUNDO CONSENSO BRASILEIRO SOBRE DISLIPIDEMIAS. Detecção, avaliação e tratamento. Arquivos Brasileiros de Cardiologia. 63(supl.): 1, 1996.

21. SILVA, C.A. e LIMA, W.C. Efeito Benéfico do Exercício Físico no Controle Metabólico do Diabetes Mellitus Tipo 2 à Curto Prazo. Arquivos Brasileiros de Endocrinologia \& Metabologia. V. 46, n. 5 Outubro 2002
22. IV DIRETRIZ BRASILEIRA SOBRE DISLIPIDEMIAS E PREVENÇÃO DA ATEROSCLEOROSE. Departamento de Aterosclerose da Sociedade Brasileira de Cardiologia. Arquivo Brasileiro de Cardiologia, Rio de Janeiro. 88, suplemento I, abril, 2007.

23. FARIA, E.C.; DALPINO, F.B.; TAKATA. Lípides e lipoproteínas séricos em crianças e adolescentes ambulatórias de um hospital universitário público. Revista Paulista de Pediatria, São Paulo. V. 26, n. 1, p. 54-58, 2008.

24. LYRA, R. et al. Prevenção do Diabetes Mellitus Tipo 2. Arquivos Brasileiros de Endocrinologia \& Metabologia. V. 50, n. 2 Abril 2006.

\section{Endereço para Correspondência:}

Renata Pereira

20renat@gmail.com

Rua $\mathrm{H}$, número 129, complemento 202

Bairro Jardim Ponte Alta - Volta Redonda - RJ

CEP: $27267-560$

Informações bibliográficas: 